University for Business and Technology in Kosovo

UBT Knowledge Center

Oct 27th, 3:15 PM - 4:45 PM

\title{
Cloud oriented Additive Technology use for Fast Prototype Development
}

\author{
Betim Shabani \\ SS. Cyril and Methodius University, betim.shabani@hotmail.com \\ Vladimir Dukovski \\ SS. Cyril and Methodius University, vladimir.dukovski@mf.edu.mk
}

Follow this and additional works at: https://knowledgecenter.ubt-uni.net/conference

Part of the Robotics Commons

\section{Recommended Citation}

Shabani, Betim and Dukovski, Vladimir, "Cloud oriented Additive Technology use for Fast Prototype Development" (2018). UBT International Conference. 335.

https://knowledgecenter.ubt-uni.net/conference/2018/all-events/335

This Event is brought to you for free and open access by the Publication and Journals at UBT Knowledge Center. It has been accepted for inclusion in UBT International Conference by an authorized administrator of UBT Knowledge Center. For more information, please contact knowledge.center@ubt-uni.net. 


\title{
Cloud oriented Additive Technology use for Fast Prototype Development
}

\author{
Betim Shabani ${ }^{1}$, Vladimir Dukovski ${ }^{2}$ \\ ${ }^{1} \mathrm{PhD}$ Student, Faculty of Mechanical Engineering, SS. Cyril and Methodius University, P.O. \\ Box 464, MK-1001, Skopje, Republic of Macedonia \\ betim.shabani@hotmail.com \\ ${ }^{2}$ Faculty of Mechanical Engineering, SS. Cyril and Methodius University, P.O. Box 464, \\ MK-1001, Skopje, Republic of Macedonia \\ vladimir.dukovski@mf.edu.mk
}

\begin{abstract}
Digitalization has already affected every segment of the industry and especially manufacturing. Based on market demands that are more specific and faster than ever, there is a need to use some of the online platform packages called Cloud Manufacturing (CM). CM works only with CAD models in general or STL models that is the official format of Additive Technologies (AT). On the other hand, there is a rapid increase in the measurement aspects via non-contact forms (3D scanners) where their data are stored in various digital formats: IGES, OBJ, PLY, etc. Those formats can be processed step by step and follow the full path to Reverse Engineering (RE). In this paper there will be discussed the possibility of implementing Cloud Additive Technology (CAT) for Fast Prototype Development (FPD) by analyzing the current situation, barriers during incorporation RE and AT, security and technicaleconomical aspects.
\end{abstract}

Keywords: Cloud Manufacturing (CM), Additive Technologies (AT), Reverse Engineering (RE), Cloud Additive Technology (CAT), Fast Prototype Development (FPD) 


\section{Introduction}

Based on the past years, Cloud Manufacturing (CM) was just a paradigm and now it is a potential reality that has wide reach all over the world. Undoubtedly this would not be possible without the notion of the Internet of Things (IoT) and other technologies that complement the wide network for access to information by attaching various production services [1]. Today, manufacturing is involved in all sectors as enabling design, maintenance, development and all other connections to a final product. Now CM is considered as an intelligent model for performing various applications [2].

On the other hand, manufacturing techniques have advanced greatly by achieving a high degree of industrial aspects and especially Additive Technology (AT) which is already known for its functionality compared to the traditional subtractive manufacturing. First of all, as the first step is CAD format of the model that is selected for production and this model digitally converts into the language of $3 \mathrm{D}$ Printers. These CAD models are most often designed or remodeled from existing parts through Reverse Engineering (RE) technology that is a highly applicable form. These two AT and RE technologies are very important for incorporation as a line in CM, which will actually lead us to Fast Prototype Development (FPD) that is also the subject of discussion in this paper.

\section{Literature Review}

Over the last few years many different authors have conducted research in the CM and AT in general and each has an analogy that makes them interconnected.

Lin Zhang et. al (2012) explained that CM will become a typical representative of the advanced manufacturing modes in the next period, whose implementation needs more works under the demand of applications and the development of technologies. The studies and applications of CM technologies will further improve the "networking, intelligent and service' of manufacturing and then promote the manufacturing information to a new level [3].

M. Mourad et. al (2016) presented a theoretical framework that is modeled to address interoperability within the CM components as each component was conceptualized and related functions were explicitly analyzed. He predicts some future work that will focus on: development of prototype software and design of industrially inspired experiment cases [4].

Yuanbin Wang et. al (2017) studied Design for Additive Manufacturing (DfAM) in cloud platform and proposed to help the customers access both hard resources and soft resources of AM [5].

\section{Cloud Additive Technology for Fast Prototype Development}

Continuous market demands for fast and cheap production have led many manufacturing companies to change their approach and meet the real demands. Sometimes the need for online access is also due to the lack of adequate equipment 
for the realization of practical parts because as we know, to produce parts that are applied in industry we need tools, machines and professional equipments which are very expensive. As one of the newest technologies that is emerging, is called Additive Technologies (AT) that is a great opportunity for easier access to the broader Internet Network due to its advantages. To use AT we need CAD model and that model needs to be sent in proper machine but before, it should be converted into several forms for AT. In some cases, original parts need reconstruction because of their application or sometimes because of damages, so in this situations CAD models are missed and for those equipments, like $3 \mathrm{D}$ scanners, it is very useful to get the data. Their output format is Point Clouds (PC). To follow the line from PC to CAD model, we use Reverse Engineering (RE) as an opportunity for remodeling for new reproduction. Even for RE, there are some online opportunities that serve to get models in some digital formats.

\subsection{RE Cloud platform}

$\mathrm{RE}$ is the science of taking the existing physical model and reproducing its surface geometry in three-dimensional (3D) data file on a computer-aided (CAD) system. The generic process of RE is a three-phase process. The three phases are: scanning, point processing and application of specific geometric model development. Each of these phases are important. Equipments and softwares must be professional and accurate.

Nowadays, there are some companies that provide 3D scanning services and have some of their scanned models (Figure 1) on their websites as: Artec 3D [6], Direct Dimensions [7], Laser Design [8], etc.

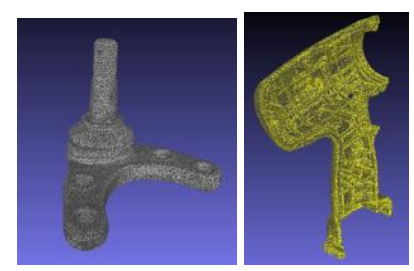

Fig. 1. Point cloud models: a) Ball joint, b) Makita case [6]

\subsection{AT Cloud platform}

The Cloud Additive Technology platform is a very effective way to meet customer requirements. The importance is the easy access and the choice of different AT technologies. The platform is built in three segments under other conditions which must be completed to the final form.

Firs one, import of the CAD model [9]. The CAD model must be converted to STL as the official AM format. The advantage is in the choice of various shapes and complex forms that make it very useful and applicable technology for production.

Second one, Automated Analysis and Assessment of Geometry [9] is presented in four steps: checking of manufacturing restriction and design guidelines, quotation costing, 
assessment of potential, part screening and selection. This segment is foreseen for technical and economic analysis of the part which is in the process for completion.

Third one, in Cloud-based Order processing [9] there are four steps presented: order acceptance, management of manufacturing inquires and orders, customer specific online parts catalog and messaging systems. This segment belongs to the management sector after the completion of the previous phases.

Once these three main segments (first, second and third) are done, then order will be switch to the next steps that completes the entire process (Figure 2).

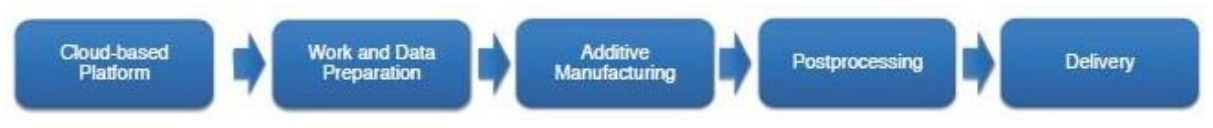

Fig. 2. CAT platform [9]

\subsection{Techno-economic aspects of CAT (case studies)}

Many CM [10] platforms are now all over the world and recently they are also used in AT. Some of them have their advantages and disadvantages but in general beside them the clients make the selection for they need and always try to find the way with accurate and the cheapest solution. At the moment, the level of discussion and requirements are in a non-industrial character, knowing that knowledge about engineering is not presented in most of the users and especially in AT which is a relatively new field. What is very important in CAT platforms is that day-to-day they are growing up. According to this, in this section there will be presented two platforms as case studies: 3D Hubs and Sculpteo.

\section{D Hubs (case study 1)}

Selected parts that will be analyzed are from industrial field, where one is used in the textile sector (Figure 3) while the other one in water industry (Figure 4). The presented parts were produced through the 3D Hubs [11] cloud platform. The reason for choosing this platform was that it enables more choices of materials, technologies and it is related with different companies that provide AT services.

As in Figure 5, we first have to choose "Upload your part" where one of the STL, STEP or IGES formats should be selected and then needs to be selected the type of material through "Select a material" (Figure 6) where the technology is presented like FDM, SLA, SLS, etc. and finally is the selection of the service with which you want to run the required part through option "Select a 3D printing service" (Figure 7).

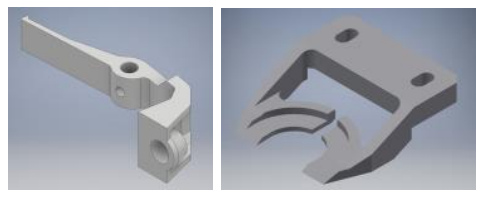


Fig. 3. Lever Fig. 4. Holder

Those are some of technical aspects and the procedure continues with the completion of other data such as: delivery method, description, payment, etc.
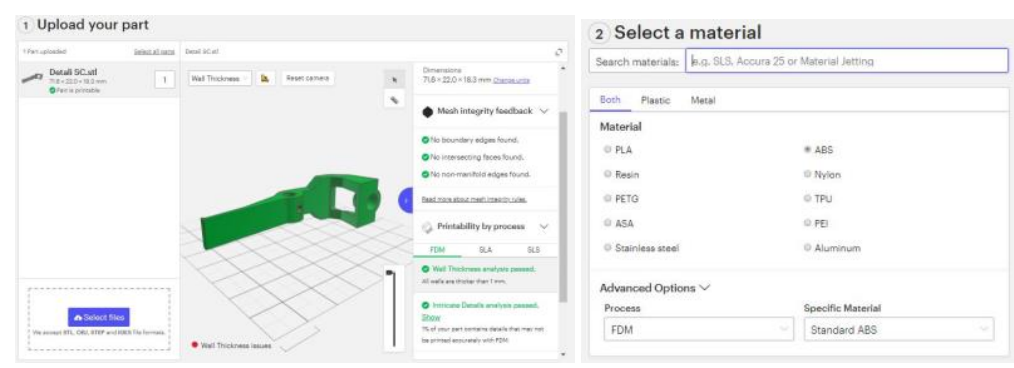

Fig. 5. Option (upload your part) [11] Fig. 6. Option (select a material) [11]

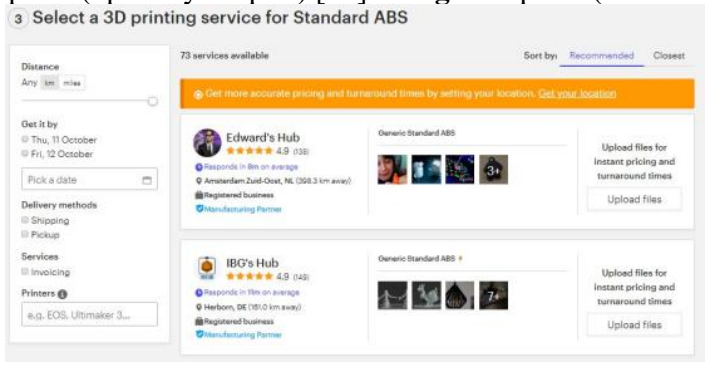

Fig.7. Option (select a 3D printing service) [11]

We all know that besides the technical factors and the aspects that have to do with the functionality the economic factor, it is very important to include the price together with time of delivery. In our case we have made requests as a test for production of parts (lever and holder) from 1 to 999 pieces. We can see the values through the diagrams in Figure 8 and Figure 9.

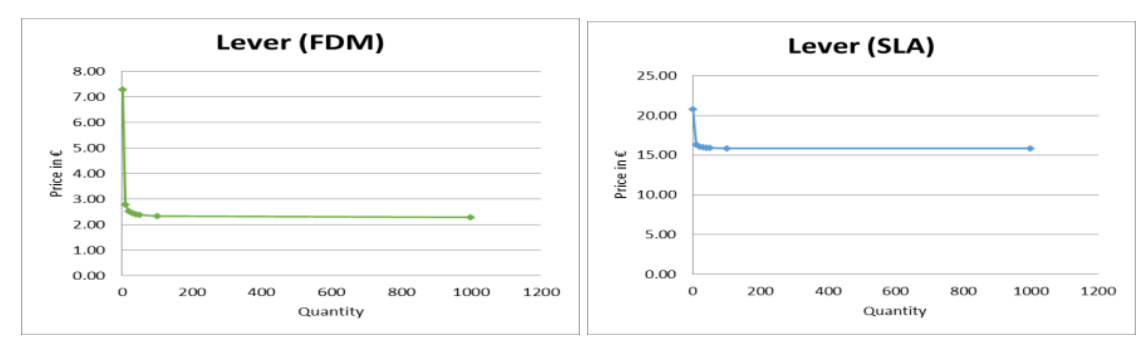

Fig. 8. Price-quantity diagram of Lever (FDM and SLA) 


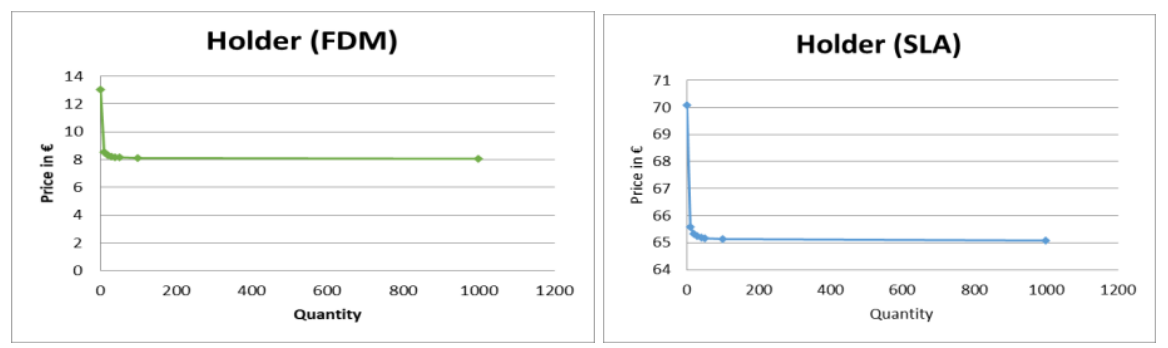

Fig. 9. Price-quantity diagram of Holder (FDM and SLA)

Fused Deposition Modeling (FDM) [12] and Stereo lithography (SLA) [13] are two Additive Technologies that are presented to be analyzed as shown in the diagrams. The reason for their selection was because they are technologies with different materials, operations and capacity. In this situation, it is important the form of price calculation and the stability of the price after increasing the quantity in two models that proves that these technologies in the coming years will be ready for mass production.

\section{Sculpteo (case study 2)}

Sculpteo [14] cloud platform is different from the previous one because the parts that are presented for production are realized by the company itself (in their factory) so it is not a platform that is related with other companies that possess 3D Printers. In some cases, this is good for parts accuracy and quality preservation but in some other cases there is a lack of access to several types of AT. Two models selected for analysis were presented in section 3.1 (point cloud models). Ball joint (Figure 10) and Makita case (Figure 11) are industrial parts with different applications. The reason for selecting them was their geometry, possibility to manufacture with different AT processes and total time analysis from $3 \mathrm{D}$ scanning to Cloud processing.

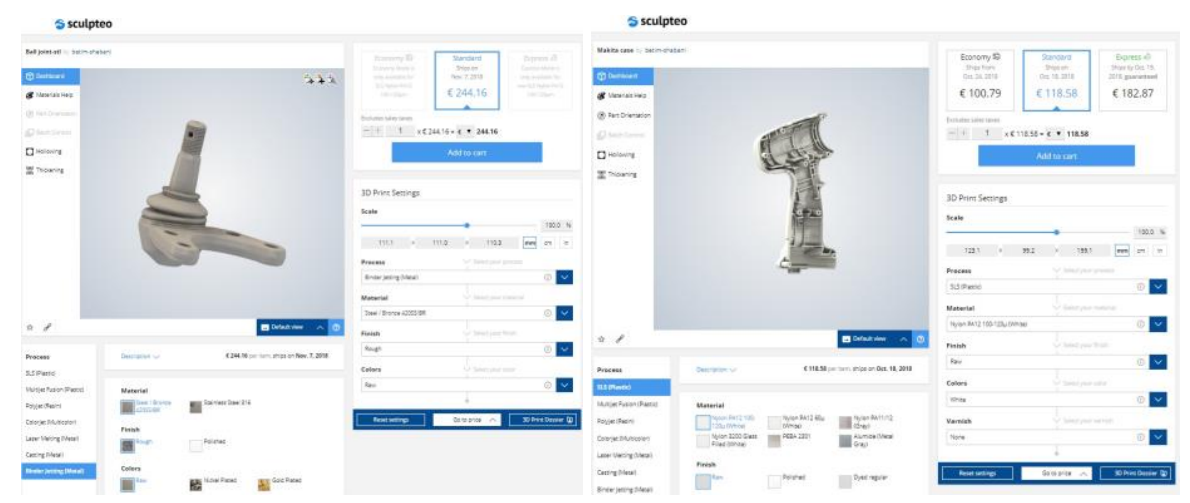

Fig. 10. Ball joint [14]

Fig. 11. Makita case [14] 
Based on the Sculpteo platform, it first needs to be uploaded the model in STL or OBJ format (for better results), that needs a relatively short time to upload the model to the database. Next steps are: importing file, file analysis, counting parts, checking manufacturability and finally file generating.

Table 1. Time processing of selected parts

\begin{tabular}{clcc}
\hline & & Ball Joint & Makita Case \\
\hline \multirow{2}{*}{ Part } & Size & $47.9 \mathrm{MB}$ & $95.3 \mathrm{MB}$ \\
& Dimensions & $111.1 \times 111 \times 110.3$ & $123.1 \times 99.2 \times 199.1$ \\
\hline \multirow{2}{*}{ Scan } & Scanning time & $3 \mathrm{~min}$ & $15 \mathrm{~min}$ \\
& Processing time & $25 \mathrm{~min}$ & $40 \mathrm{~min}$ \\
\hline \multirow{2}{*}{ Cloud } & Upload time & $1 \mathrm{~min} 59 \mathrm{sec}$ & $3 \mathrm{~min} 55 \mathrm{sec}$ \\
& Processing time & $15 \mathrm{sec}$ & $55 \mathrm{sec}$ \\
\hline
\end{tabular}

According to the Table 1, it is noted that the time required to complete the Ball Joint is 30 minutes and 14 seconds while for Makita Case is 59 minutes and 50 seconds. In general, this shows that the Scanning process and Cloud platforms are very effective in time saving while keeping the accuracy.

\subsection{Proposed solution for FPD by using RE and AT}

The need for integrated RE [15, 16] and AT in one big Cloud Manufacturing Environment is crucial. Those two technologies present complete line from the beginning to the final product. Their use will be very effective especially for cases when the original parts exist and need engineering reconstructions or they are damaged and must be reproduced. Based on the previous sections presented in this paper and the line that reach to FPD [17, 18], we propose scheme (Figure 12) that includes 3D scanning [19, 20], RE and CAT. 


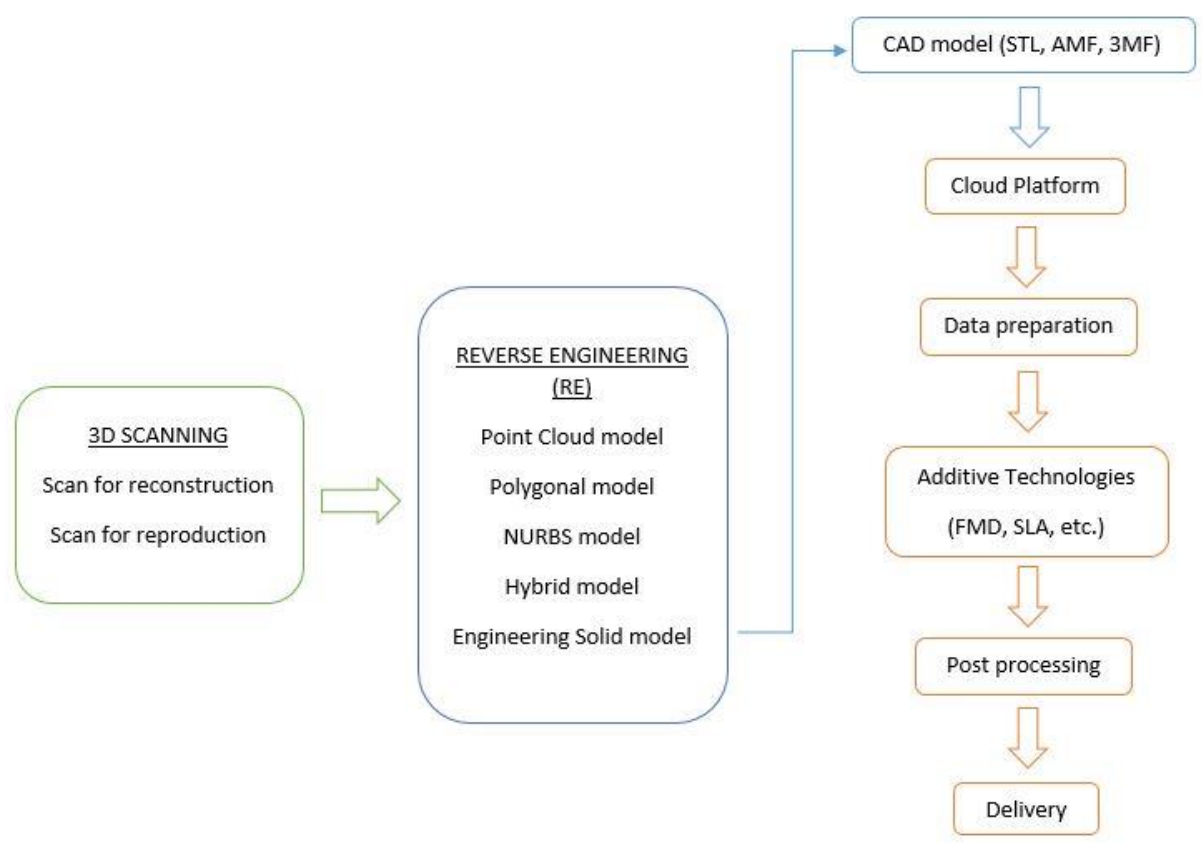

Fig. 12. Scheme for Fast Prototype Development by using 3DS, RE and CAT

What we see in Figure 12 is a complete digital form from measurement to delivery, which greatly affects shortening time according to Table 1 and also gives the opportunity for any correction between steps. It is a great opportunity for these platforms to be present in the market in the future.

\section{Conclusion}

This paper presents the current approach and orientation of production in general, specifically that of AT. The focus of the paper has been on the possibility of interconnection between RE and AT as a digital line to finally end up with a CAT platform that will lead us towards the FPD. In two practical case studies we see the workflow in online platform and understood CAT aspects.

According to quantity-price diagrams (case study 1), we see a decrease in the price when the amount increases, which indicates that this technology is moving towards mass production. On the other hand, as seen from the table (case study 2), we can conclude that even parts with complex geometry can be produced quickly.

An important topic for the future is the possibility of practical realization of the cloud connection between $3 \mathrm{D}$ scanners, $\mathrm{RE}$ and $\mathrm{AT}$ as a real opportunity for parts that are in the market that need to be changed, improved and reproduced. 


\section{References}

1. Mohamed MOURAD, Aydin NASSEHI, Stephen NEWMAN, Dirk SCHAEFER: C-MARSABM: A Deployment Approach for Cloud Manufacturing. Advances in Manufacturing Technology XXXI, (2017) 213-218

2. Lei Rena, Lin Zhang, Fei Tao, Chun Zhao, Xudong Chai, Xinpei Zhao: Cloud manufacturing: from concept to practice. Enterprise Information Systems, Vol. 9, No. 2, Taylor \& Francis, (2013) 186-209

3. Lin Zhang, Yongliang Luo, Fei Tao, Bo Hu Li, Lei Ren, Xuesong Zhang, Hua Guo, Ying Cheng, Anrui $\mathrm{Hu}$, Yongkui Liu: Cloud manufacturing: a new manufacturing paradigm. Enterprise Information Systems, Vol. 8, No. 2, Taylor \& Francis, (2012) 167-187

4. M. Mourad, A. Nassehi, D. Schaefer: Interoperability as a key enabler for manufacturing in the cloud. Procedia CIRP 52 (2016) $30-34$

5. Yuanbin Wang, Robert Blache, Xun Xu: DESIGN FOR ADDITIVE MANUFACTURING IN THE CLOUD PLATFORM. MSEC2017, Los Angeles, CA, USA (2017)

6. Artec 3D: Industrial design and manufacturing 3D models. https://www.artec3d.com/3dmodels/ball-joint

7. Direct Dimensions: Sample 3D Models. http://www.directdimensions.com

8. Laser Design: Sample Files. https://www.laserdesign.com/sample-files/

9. Jan-Peer Rudolph, Claus Emmelmann: A Cloud-based Platform for Automated Order Processing in Additive Manufacturing. Procedia CIRP 63 (2017) 412 - 417

10. FelixW. Baumann, Dieter Roller: Additive Manufacturing, Cloud-Based 3D Printing and Associated Services-Overview. J. Manuf. Mater. Process. 2017, 1

11. 3D HUBS: 3D Printing. https://www.3dhubs.com/3d-printing

12. P. Chennakesava, Y. Shivraj Narayan: Fused Deposition Modeling - Insights. International Conference on Advances in Design and Manufacturing (ICAD\&M'14), (2014)

13. Benay Sager, David Rosen: STEREOLITHOGRAPHY PROCESS RESOLUTION, (2015)

14. Sculpteo. 3D Printing. https://www.sculpteo.com/en/

15. Kwan H. Lee, H. Woo: Direct integration of reverse engineering and rapid prototyping. Computers\&Industrial Engineering 38, (2000) 21-38

16. V. K. Kumbhar, P. M. Pandey, P.V. M. Rao: Improved intermediate point curve model for integrated reverse engineering and rapid prototyping. Int J Adv Manuf Technology, (2007)

17. Lalit Kumar, Vineet kumar, Abid Haleem: Rapid Prototyping Technology for New Product Development. IJISET, Vol. 3 Issue 1, (2016)

18. S. O. O N U H, Y. Y. Y U S U F: Rapid prototyping technology: applications and benefits for rapid product development. Journal of Intelligent Manufacturing, (1999) 301-311

19. Creaform: AN INTRODUCTION TO 3D SCANNING, Creaform Ebook Series, (2015)

20. LMI TECHNOLOGIES: A Simple Guide To Understanding 3D Scanning Technologies, (2013) 